\title{
ESTRATÉGIAS DIDÁTICAS NA EDUCAÇÃO DIGITAL MOOC RRI
}

\author{
Katia Ethiénne Esteves dos Santos 1 \\ Patrícia Lupion Torres 2 \\ Pierre Narcisse Chery ${ }^{3}$
}

\begin{abstract}
RESUMO
As transformações acontecem de forma vertiginosa nos últimos anos em nossa sociedade, impulsionando os diferentes processos e as relações entre grupos sociais. A educação digital possibilita o acesso à informação e ao conhecimento em diferentes espaços, contextos e temporalidades, tanto em ambientes formais de educação quanto não formais, na perspectiva da aprendizagem on-life. Este cenário passa a demandar a necessidade de que as pessoas estejam formadas para a gestão do conhecimento que envolve a aprendizagem ao longo da vida, o ser autônomo, o saber, o pesquisar, o refletir, o analisar e criar soluções possíveis. Na perspectiva de mudança nas propostas didático-pedagógicas-tecnológicas verifica-se a premissa de utilizar estratégias didáticas diferenciadas. Para esta reflexão, os autores Santos (2018), Okada (2016), Torres (2017) e Kowalski (2018) entre outros dão sustentação para a discussão referente a valorização efetiva dos estudantes na construção do conhecimento e no desenvolvimento de investigadores, com foco na proposta de Pesquisa e Inovação Responsáveis (Responsible Research and Innovation - RRI) oportunizando a estes pesquisar, refletir, experimentar e criar, partindo de situações-problema reais. Este artigo resulta de uma investigação realizada com um grupo de estudos do mestrado e doutorado em Educação e propõe-se a refletir sobre o processo de construção de um curso a distância (MOOC) para a formação de professores na utilização da proposta de RRI. O curso busca valorizar a pesquisa e a inovação, estruturado em cinco módulos que envolvem uma ambientação inicial e as habilidades de investigação científica por meio do adotar, do adaptar, do transformar e do aplicar.
\end{abstract}

Palavras-chave: RRI. Educação Digital. Pesquisa.

\section{TEACHING STRATEGIES IN DIGITAL EDUCATION}

\footnotetext{
${ }^{1}$ Doutora em Educação, Professora da Pontifícia Universidade Católica do Paraná - PUCPR, Curitiba, Paraná, Brasil. ORCID ID: http://orcid.org/0000-0003-4582-1139 E-mail:katia.esteves@pucpr.br

2 Doutorado em Engenharia de Produção (UFSC), Professora da Pontifícia Universidade Católica do Paraná - PUCPR, Curitiba, Paraná, Brasil. ORCID ID: http://orcid.org/0000-0003-2122-1526 E-mail: patricia.lupion@pucpr.br

${ }^{3}$ Mestre em Educação, Doutorando Pontifícia Universidade Católica do Paraná - PUCPR, Curitiba, Paraná, Brasil. ORCID ID: http://orcid.org/0000-0002-3168-0526 E-mail: narcissechery7@gmail.com
} 


\title{
MOOC RRI
}

\begin{abstract}
The transformations have taken place in a dizzying way in recent years in our society, boosting the different processes and the relationships between social groups. Digital education enables access to information and knowledge in different spaces, contexts and temporalities, both in formal and non-formal educational environments, from the perspective of on-life learning. This scenario starts to demand the need for people to be trained in the management of knowledge that involves lifelong learning, being autonomous, knowing, researching, reflecting, analyzing and creating possible solutions. In the perspective of change in the didacticpedagogical-technological proposals, the premise of using different didactic strategies is verified. For this reflection, the authors Santos (2018), Okada (2016), Torres (2017) and Kowalski (2018), among others, support the discussion regarding the effective valorization of students in the construction of knowledge and the development of researchers, focusing in the Responsible Research and Innovation (RRI) proposal, giving them the opportunity to research, reflect, experiment and create, based on real problem situations. This article is the result of an investigation carried out with a group of master's and doctoral studies and is willing to reflect on the process of building a distance course (MOOC) for the training of teachers in the use of the RRI proposal. The course seeks to value research and innovation, structured in five modules that involve an initial setting and scientific research skills through adopting, adapting, transforming and applying.
\end{abstract}

Keywords: RRI. Digital Education. Search

\section{ESTRATEGIAS DE ENSEÑANZA EN EDUCACIÓN DIGITAL}

MOOC RRI

\section{RESUMEN}

Las transformaciones han tenido lugar de manera vertiginosa en los últimos años en nuestra sociedad, impulsando los diferentes procesos y las relaciones entre los grupos sociales. La educación digital permite el acceso a la información y al conocimiento en diferentes espacios, contextos y temporalidades, tanto en entornos educativos formales como no formales, en la perspectiva del aprendizaje en la vida. Este escenario comienza a exigir la necesidad de capacitar a las personas en la gestión del conocimiento que implica el aprendizaje permanente, ser autónomo, conocer, investigar, reflexionar, analizar y crear posibles soluciones. En la perspectiva del cambio en las propuestas didáctico-pedagógicotecnológicas, se verifica la premisa de utilizar diferentes estrategias didácticas. Para esta reflexión, los autores Santos (2018), Okada (2016), Torres (2017) y Kowalski (2018), entre otros, apoyan la discusión sobre la valorización efectiva de los estudiantes en la construcción del conocimiento y el desarrollo de investigadores, centrándose en la propuesta de Investigación e Innovación Responsable (IRR), dándoles la oportunidad de investigar, reflexionar, experimentar y crear, basándose en situaciones problemáticas reales. Este artículo es el resultado de una investigación 
realizada con un grupo de estudios de maestría y doctorado y está dispuesto a reflexionar sobre el proceso de construcción de un curso a distancia (MOOC) para la capacitación de maestros en el uso de la propuesta de RRI. El curso busca valorar la investigación y la innovación, estructurado en cinco módulos que involucran un entorno inicial y habilidades de investigación científica a través de la adopción, adaptación, transformación y aplicación.

Palabras clave: RRI. Educación digital. Investigación.

\section{INTRODUÇÃO}

A importância das mudanças atuais vai muito além das transformações tecnológicas, pois essas atingem áreas sociais, culturais, políticas e ambientais. A ampliação do universo das habilidades cognitivas e socioemocionais necessárias para o desenvolvimento individual neste século ultrapassa as estratégias de resolução de problemas.

Fruto da globalização e da revolução tecnológica, as transformações atingem as áreas da produção, do consumo, das negociações e do mundo do trabalho, impactando de forma marcante nas mudanças essenciais no dia a dia das pessoas, como o vestir, o alimentar-se, o trabalhar, o relacionarse e até o cuidado com a saúde.

As tecnologias e seus recursos vêm transformando formas de produzir, assim como os meios de consumo, o que tem afetado diretamente a educação de crianças, jovens e adultos, oferecendo caminhos para que possam tornar-se cidadãos críticos e atuantes, capazes de analisar o mundo ao seu redor, fazer escolhas e ser feliz.

Com base nisso, os agentes sociais reelaboram formas de relacionamento com o espaço e com o tempo, apoiados pelas conexões em rede que permitem a interação, a comunicação, a aprendizagem e as mudanças no modo como cada um se constitui, possibilitando uma modelagem na associação que se estabelece. 
As tecnologias e as inovações abrem espaço para que ocorram inúmeras transposições de informações e conhecimentos entre sujeitos oriundos de espaços e culturas diferentes.

O relatório europeu The education and training monitor (EUROPEAN COMISSION, 2017, p. 88) indica a existência de déficit pedagógico em relação ao que se projeta como futuro sendo construído no presente, portanto, "não é mais suficiente equipar os jovens com um conjunto fixo de conhecimentos; eles também precisam desenvolver a capacidade de lidar com a mudança".

Entendemos que para este tempo de educação digital sejam cruciais a criatividade, a comunicação, a inteligência emocional e as competências transversais, que constituirão a qualificação-base para o sucesso no mundo do trabalho e na vida dos indivíduos. Assim, esta pesquisa objetiva apresentar percepções levantadas de forma qualitativa, e foi aplicada com os sujeitos participantes do grupo de pesquisa que está apoiando a construção da proposta do curso MOOC-RRI e que pretende divulgar as ideias de RRI.

\section{Educação digital}

Frente ao universo apresentado, construir um espaço social e educacional, no qual sejam levados em conta as transformações digitais, os desafios sociais e as transformações culturais, passa a ser relevante na constituição de qualquer proposta que objetive o desenvolvimento de competências e na formação de cidadãos.

Com relação a educação, tivemos momentos diferenciados iniciando na classificada Educação 1.0 (Fava, 2012), que se constituiu até meados do século XVIII, tendo como característica principal o ensino de um para muitos de forma tradicional, com recursos como livros, lousa, giz e objetos para 
atividades recreativas, em um ambiente físico determinado como sala de aula.

É notório que a grande consolidação do sistema educacional aconteceu por meio das demandas impostas pela Revolução Industrial, na qual os trabalhadores tiveram que desenvolver atividades repetitivas, sequenciadas, segmentadas e muito bem definidas. A escola era a responsável por formar esses cidadãos para as necessidades do trabalho.

Até meados do século XX, com a evolução nas exigências no mundo do trabalho, impulsionadas pelos reflexos da Revolução Industrial, em alguns momentos na sala de aula (espaço físico que se mantém), o professor oferecia a oportunidade de os estudantes realizarem alguma atividade em duplas, ou grupos. Iniciou ali a utilização de algumas tecnologias educacionais (hardwares e softwares) nos laboratórios de informática montados nas escolas, ampliando o espaço de atuação oferecido para o processo de ensino-aprendizagem. Podemos classificar como Educação 2.0, na perspectiva abordada nesta reflexão, como cita (Fava, 2012).

Ao oferecer espaços de atuação e colaboração que podem ser físicos, ou virtuais, nos quais os estudantes interagem com seus pares, ampliando as oportunidades de aprendizagem, temos a Educação 3.0. Nesse sentido, a educação híbrida (Santos, 2018), sendo uma parte das atividades on-line e uma parte presencial, e a educação a distância ampliam os espaços de aprendizagem, oferecendo oportunidades diferenciadas de desenvolvimento individual e coletivo. Ainda, espaços diferenciados de aprendizagens são cada vez mais observados, derrubando os muros da escola.

Ao envolver estudantes na perspectiva da aprendizagem ao longo da vida, em projetos de construção coletiva do conhecimento, na perspectiva de resolução de problemas e de desenvolvimento socioemocionais, temos a chamada Educação 4.0. 
Esta era digital oferece uma experiência não linear, conectada, multidisciplinar e exponencialmente imprevisível, e apresenta desafios constantes que impulsionam uma visão diferenciada para as propostas educacionais.

Dessa forma, torna-se relevante a utilização de espaços para aprendizagem criativa, como o Maker, em uma perspectiva STEAM Science, Technology, Engineering, Arts e Mathematics -, reunindo conhecimentos de Artes, Ciências, Tecnologia, Engenharia e Matemática, que podem ampliar as oportunidades de ensinar e de aprender, além de desenvolver cidadãos capazes de resolver problemas e de cuidar do contexto em que vive.

Ao pensar os constantes desafios do século, objetivar a investigação, a descoberta, as conexões entre os agentes envolvidos, a criação e a reflexão possibilitam a mudança efetiva das relações com o aprender e o ensinar.

As tecnologias fazem cada vez mais parte do nosso cotidiano, com destaque para a inteligência artificial e as inovações constantes que permitem personalização e interação entre os envolvidos. Entendemos como fundamental propor atividades que possibilitem o desenvolvimento da criatividade, da curiosidade e do pensamento crítico como forma de preparar os estudantes para um futuro de mudanças mais efetivas.

\section{Conceitos RRI}

No contexto de promoção da ciência e da tecnologia de maneira desejável, parece ser indispensável pensar nos processos de investigação e inovação e, portanto, nas necessidades e valores das sociedades que os sustentam. Nesse caso, é mais que evidente a importância de envolver toda a sociedade nas decisões relacionadas com o desenvolvimento da ciência e da tecnologia para poder contribuir no crescimento social inteligente, sustentável e inclusivo, criando um projeto de transformação efetiva. 
Beltran et al (2017, p.1041) entendem que a Investigação e Inovação Responsáveis (RRI - Responsable Research and Innovation) representa um conceito emergente que está crescendo de forma espantosa na União Europa (EU). A partir de 2001, essa proposta foi incorporada em diferentes países como um plano de ação para o desenvolvimento da "ciência e a sociedade" com a finalidade de estabelecer uma estratégia comum para melhorar a relação entre a ciência e a cidadania. Desde então, a RRI tem alcançado presença mais significativa nas conferências europeias e tem sido usada em alguns casos para definir as linhas de investigação financiadas.

Para o Comité Économique et Social Européen - CESE (2016), a expressão "Recherche et innovation responsables" (RIR) se explica como um processo contínuo que busca colocar a investigação e a inovação no eixo dos valores, das necessidades e das expectativas da sociedade.

De maneira geral, entendemos que Pesquisa e Inovação Responsáveis (RRI) implica na participação de todos os atores, desde pessoas da comunidade investigativa até as instituições e os governos, utilizando metodologias inclusivas e participativas durante todas as etapas dos processos de pesquisa e inovação. Além disso, em todos os níveis de governança de investigação e inovação (do estabelecimento da agenda ao desenho, à implementação e à avaliação).

Fialho, Reis e Marques (2016) citam que o termo Investigação e Inovação Responsáveis é muito recente e está em plena evolução. O processo transparente $e$ interativo que permite aos atores sociais e inovadores, de forma mútua e responsável com foco na aceitabilidade, sustentabilidade e expectativa social do processo de inovação e dos seus produtos de maneira a facilitar adequadamente a incorporação dos avanços científicos e tecnológicos na sociedade. 
Portanto, autores pensam que o conceito de Investigação e Inovação Responsáveis pode ter um carácter muito mais amplo. Isso significa, então, para esses autores, um "cuidado do futuro, por meio de coordenação e colaboração coletiva da ciência e inovação no presente".

O pesquisador Herrera (2015, p.65) enfatiza que a RRI mostra que as inovações sociais representam novas soluções, "produtos, serviços, processos, mercados, formas de organização e modelos de negócios", que de forma simultânea cumprem com as necessidades sociais e conduzem a novas e melhoradas capacidades e relações, possibilitando o melhor uso dos recursos existentes. Essa ideia de Herrera leva a entender a importância de (RRI) Research and Innovation Resposible no setor financeiro de uma sociedade.

\section{Estratégias adotadas na RRI}

Em suas reflexões de Azinhaga, Marques e Reis (2016, p. 2036) apresentam que o autor Sutcliffe (2013) busca definir RRI em um sentido mais amplo, enumerando as dimensões que podem ser consideradas como estratégias para alcançar ou realizar o projeto. As dimensões definidas por eles são as seguintes:

a) Envolvimento - participação conjunta de investigadores, indústria e sociedade civil no processo de investigação e inovação; b) Igualdade de gênero - desbloquear todo o potencial da sociedade; c) Educação científica - educação criativa capaz de responder às necessidades futuras da sociedade; d) Ética - assegurar uma maior relevância e aceitação social dos resultados da investigação e inovação; e) Livre acesso - acesso livre e online aos resultados da investigação financiada publicamente, f) Governação - os decisores políticos são responsáveis pelo desenvolvimento de modelos harmoniosos para uma Investigação e Inovação Responsáveis.

O documento resultante do Observatori de Bioética i Dret, Universitat de Barcelona, faz a mesma consideração que Azinhaga, Marques e Reis 
(2016), descrevendo seis agendas políticas que compreendem a RRI e que devem ser consideradas em todo o processo de investigação e inovação.

Percebemos nessa reflexão que os autores se aproximam, considerando a mesma visão do projeto com estratégias diferentes.

De acordo com o Observatori de Bioética i Dret, Universitat de Barcelona, as seis agendas políticas apresentam-se na Figura 1: Seis agendas políticas.

Figura 1: Seis agendas políticas

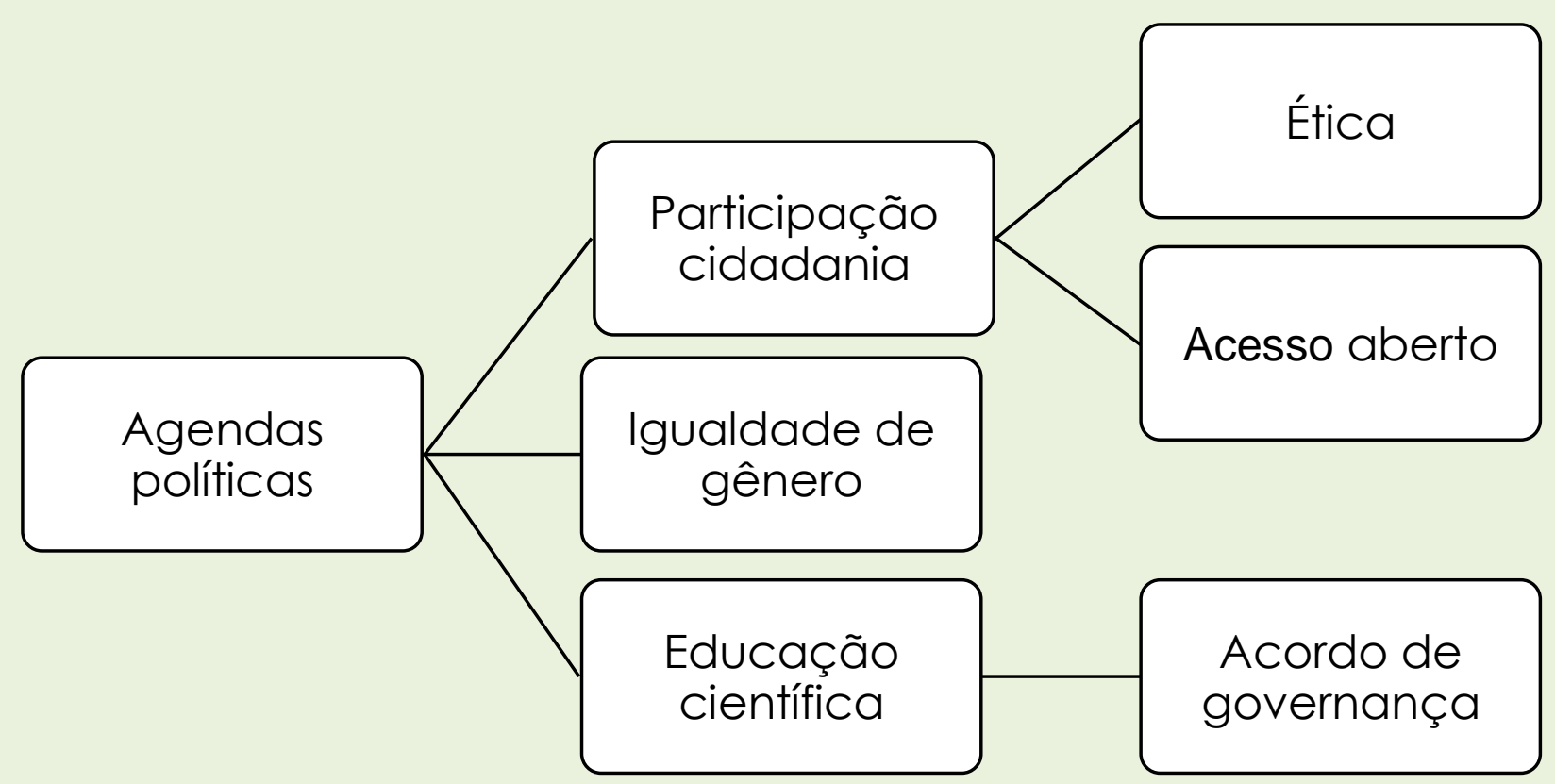

Fonte: Autores, 2019, baseados em Observatori de Bioética i Dret, Universitat de Barcelona.

Essas dimensões mencionadas por Sutcliffe (2013) representam pilares ou estratégias para concretizar o projeto formulado pela comunidade Europeia visando a sociedade em sua integralidade. Porém, é importante 
explicar e entender o contexto de cada grupo social que é abordado ou tratado pelos autores.

A pesquisa intitulada Irresistible, realizada pela Universidade Lisboa (U Lisboa) e pelo Instituto de Educação (IE) (2016), ao citar a importância das diferentes estratégias, determina que ações sejam levadas em consideração, como apresentado de forma resumida no Quadro 1: Seis estratégias, a seguir.

QUADRO 1 - Seis estratégias

\begin{tabular}{|c|c|}
\hline Participação cidadania & Igualdade de gênero \\
\hline $\begin{array}{l}\text { É importante que os conjuntos de } \\
\text { todos os atores sociais, tais como } \\
\text { investigadores, indústria, decisores } \\
\text { políticos e sociedade civil se } \\
\text { envolvam e participem no } \\
\text { desenvolvimento da RRI. Nesse } \\
\text { sentido, eles devem estar em } \\
\text { sintonia para desenvolver } \\
\text { melhores soluções, de acordo } \\
\text { com cada problema, incluindo as } \\
\text { oportunidades sociais, a fim de } \\
\text { evitar falhas das futuras } \\
\text { inovações. }\end{array}$ & $\begin{array}{l}\text { Trata-se de um envolvimento igual e } \\
\text { equilibrado de todos os atores sociais, } \\
\text { tanto do número de mulheres como de } \\
\text { homens. Nesse caso, é contundente } \\
\text { defender a ideia de aumentar o } \\
\text { número de mulheres investigadoras por } \\
\text { conta da redução. }\end{array}$ \\
\hline Educação científica & Ética \\
\hline $\begin{array}{l}\text { Ter muitos investigadores parece } \\
\text { ser uma obrigação não somente } \\
\text { para Europa, mas também para } \\
\text { todo o mundo. Desse modo, é } \\
\text { necessário aperfeiçoar o ensino } \\
\text { atual das ciências para poder } \\
\text { preparar os futuros investigadores, } \\
\text { incluindo conhecimento e } \\
\text { competências necessários no } \\
\text { sentido de uma investigação e } \\
\text { inovação responsáveis. Em efeito, } \\
\text { é essencial criar desejo de } \\
\text { aprender matemática nas } \\
\text { crianças e jovens, também das } \\
\text { ciências e da tecnologia. Assim, } \\
\text { podem os } \\
\text { investigadores do futuro com mais } \\
\text { conhecimento científico na } \\
\text { sociedade. }\end{array}$ & $\begin{array}{l}\text { Baseado nos princípios e valores, o } \\
\text { projeto de RRI deve respeitar os direitos } \\
\text { e leis feitas, porém é necessário que os } \\
\text { investigadores verifiquem baixas } \\
\text { normas e valores relacionados a uma } \\
\text { sociedade com uma vida saudável } \\
\text { garantida pela qualidade dos } \\
\text { resultados alcançados } \\
\text { investigações. }\end{array}$ \\
\hline
\end{tabular}




\begin{tabular}{|l|l|}
\hline Acesso aberto & \multicolumn{1}{|c|}{ Acordo de governança } \\
\hline A publicação dos resultados e & Em sentido concreto, os decisores \\
dados da investigação científica & políticos, sendo autoridades, devem ser \\
obtidos pela Investigação e e & envolvidos de forma harmoniosa na \\
Inovação Responsáveis deve ser & Investigação e Inovação Responsáveis, \\
acessiva a todos os atores sociais & com a finalidade de integrar todos os \\
de maneira transparente, pois a & setores da vida social. \\
pesquisa foi financiada com & Os relevantes de RRI na formação de \\
dinheiro público. Isso possibilita a & professores. \\
tomada de decisão que se & \\
fundamenta no processo & \\
investigativo científico. & \\
\hline
\end{tabular}

Fonte: Adaptado pelos autores (2020).

De forma concreta, esse novo termo Responsible Research and Innovation (RRI) adotado pela Comissão Europeia no Programa Horizon 2020 tem sido uma referência na pesquisa inovadora, abordando diferentes conceitos transversais relacionados à ciência e à inovação com a sociedade.

O programa tem impactado muitos países, causando mudanças no sistema de ensino, sobretudo no nível superior. Da mesma forma, conjugando os esforços de todos os setores da vida nacional, os professores, os estudantes, os pesquisadores ou os cientistas, os governos e as comunidades, para atingir a meta comum que é a transformação socioeconômica de uma determinada sociedade por meio da Investigação e Inovação Responsáveis.

Essa ideia inovadora pode ter cada vez mais visibilidade na América Latina, especificamente no Brasil. Vários autores destacam a importância e a necessidade da pesquisa responsável no processo de ensino-aprendizagem brasileiro, podendo o conceito ser utilizado, nas várias áreas de pesquisa, tais como saúde, educação, economia etc.

A RRI é uma competência abrangente multidimensional que abarca várias outras competências; como consequência, cada uma dessas competências interage dinamicamente com os conhecimentos, habilidades 
e atitudes de maneira a conectar valores, exigindo que o indivíduo seja capaz de aprender a aprender, aprender a fazer e aprender a ser.

A pesquisa responsável pode oferecer espaço para articulação de quatro dimensões: antecipação, reflexibilidade, inclusão e capacidade de resposta.

\section{Universo de Pesquisa}

Esta reflexão faz parte de um conjunto de ações relacionadas ao projeto de estudo de pesquisa "O uso de REA e de Tecnologias Digitais na formação de Professores na perspectiva da RRI" realizada no Programa de Pós-Graduação da PUCPR que pretende responder ao seguinte problema de pesquisa: "Como recontextualizar a abordagem da Responsabilidade, Pesquisa e Inovação na formação de professores de modo a integrar os conhecimentos curriculares envolvendo temas atuais de relevância sociocientíficas?".

Para esse projeto construiu-se um curso de formação continuada online e aberto, (Massive Open Online Course - MOOC4), que aproveita as possibilidades dos recursos tecnológicos e que se constitui como objeto de reflexão deste estudo.

Por ser uma pesquisa qualitativa, a coleta de dados e percepções foi realizada por meio de um questionário semiestruturado em um formulário do Google. A quantidade de respondentes foi de $85 \%$, sendo (14) quatorze participantes e, destes, $26,6 \%$ do sexo masculino e $71,4 \%$ do sexo feminino, como representado a seguir.

\footnotetext{
${ }^{4}$ MOOC, O que são MOOCs (Massive Open Online Courses)? Disponível em: <https://www.ufrgs.br/bibpsico/2018/02/o-que-sao-moocs-massive-open-online-courses/> Acesso em: maio 2020.
} 
Figura 2: Público da pesquisa - Sexo

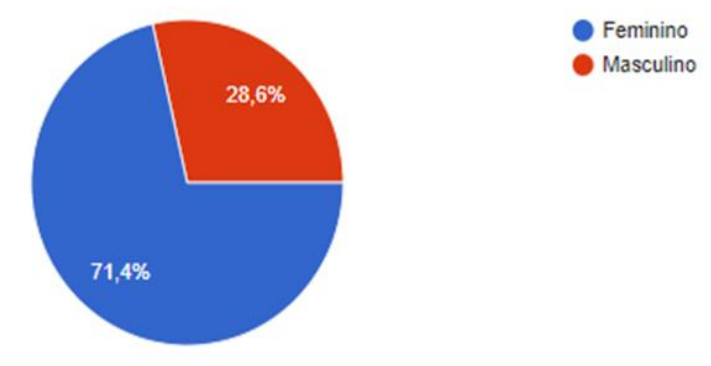

Fonte: Autores (2020).

O grupo envolvido concentra pesquisadores de diferentes faixas etárias como pode ser observado na Figura 3, o que possibilita uma gama diferenciada de olhares para o objeto em estudo.

Figura 3: Público da pesquisa - Faixa etária

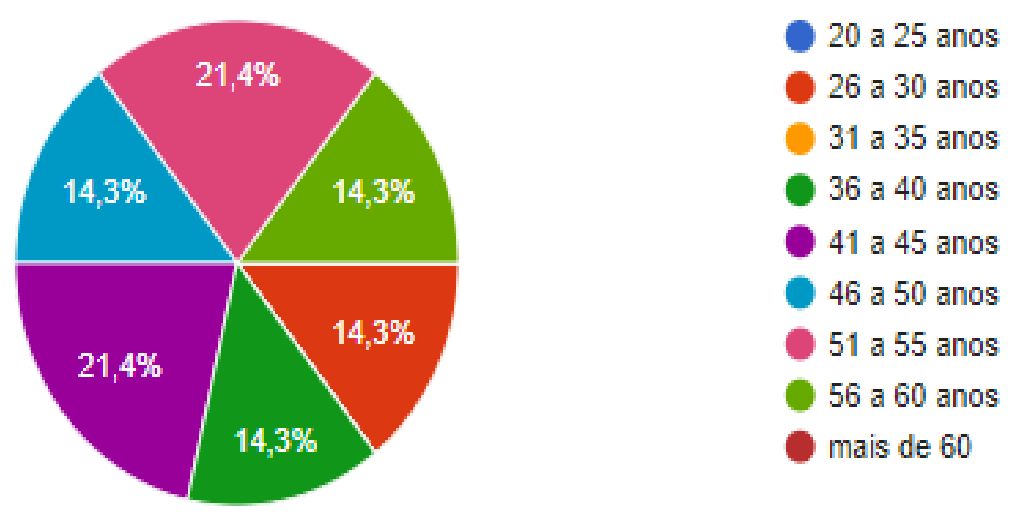

Fonte: Autores (2020).

O nível de formação dos participantes concentra-se em 57,1\% doutores, que continuam participando do grupo de pesquisa, dada a sua relevância, e nos ambientes acadêmicos, e de 35,7\% de mestres, que em 
alguns casos estão realizando seus estudos de doutoramento, além de possibilitar que estudantes com graduação (PIBIDs) também possam participar, como representado na Figura 4.

Figura 4: Público da pesquisa - Formação completa

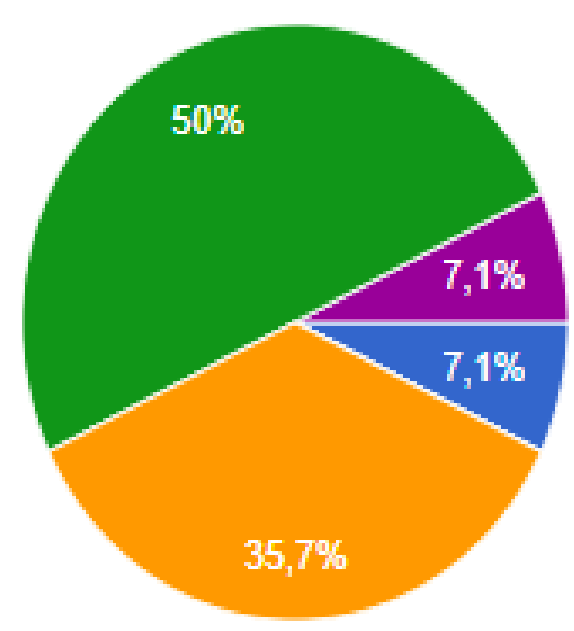

- Graduação

- Pós graduação Lato sensu

Strictu sensu mestrado

- Strictu sensu doutorado

Strictu sensu pós doutorado

Fonte: Autores (2020).

Os dados apresentados revelam 0 universo de participantes respondentes do questionário, o que permite que nessa reflexão a análise seja ampla e efetiva, principalmente por ser o tema RRI muito novo entre os estudiosos brasileiros.

\section{MOOC de RRI, percepções}

Os envolvidos na pesquisa deixaram suas percepções e registro de vivências nos apontamentos escritos nas questões específicas sobre a construção do curso em formato MOOC para a formação de professores. 
Assim, poderiam conhecer a proposta da RRI e, também, ter acesso a ferramentas e sugestões para aplicação.

O curso está estruturado em cinco módulos, como explicitado na tabela a seguir.

QUADRO 2 - Módulos do MOOC de RRI

\begin{tabular}{|l|l|}
\hline $\begin{array}{l}\text { Módulo 1 } \\
\text { Ambientação }\end{array}$ & $\begin{array}{l}\text { Oferece aos professores a contextualização sobre o MOOC } \\
\text { e sobre RRI. }\end{array}$ \\
\hline $\begin{array}{l}\text { Módulo 2 } \\
\text { Adotar }\end{array}$ & $\begin{array}{l}\text { Possibilita aos professores a opção de aprender estratégias } \\
\text { de RRI por meio de objetos de aprendizagem diferenciados. }\end{array}$ \\
\hline $\begin{array}{l}\text { Módulo 3 } \\
\text { Adaptar }\end{array}$ & $\begin{array}{l}\text { Disponibiliza um conjunto de ferramentas, atividades, jogos e } \\
\text { explicações para apoiar o processo de ensino e de } \\
\text { aprendizagem da RRI. }\end{array}$ \\
\hline $\begin{array}{l}\text { Módulo 4 } \\
\text { Transformar }\end{array}$ & $\begin{array}{l}\text { Disponibiliza aos professores a opção de acesso a novos } \\
\text { materiais de RRI, que podem ser de forma colaborativa } \\
\text { cocriados por professores e estudantes, que atuarão como } \\
\text { coconstrutores dos materiais. }\end{array}$ \\
\hline $\begin{array}{l}\text { Módulo 5 } \\
\text { Aplicar }\end{array}$ & $\begin{array}{l}\text { Recontextualizar a teoria estudada nos módulos anteriores à } \\
\text { realidade escolar e relatar a experiência vivenciada. O } \\
\text { projeto propiciará ao professor participante experiências } \\
\text { sobre como os conceitos da ciência podem ser entendidos } \\
\text { com base em seu contexto e de suas implicações para a } \\
\text { sociedade. A proposta é que cada professor apresente uma } \\
\text { atividade final, preenchendo um formulário on-line das } \\
\text { atividades realizadas. }\end{array}$ \\
\hline
\end{tabular}

Fonte: Autores (2020).

Os agentes da pesquisa participaram em seus grupos do desenvolvimento de pelo menos um módulo do curso, como revela a Figura 5, a seguir. 
Figura 5: Participantes no desenvolvimento dos módulos do curso

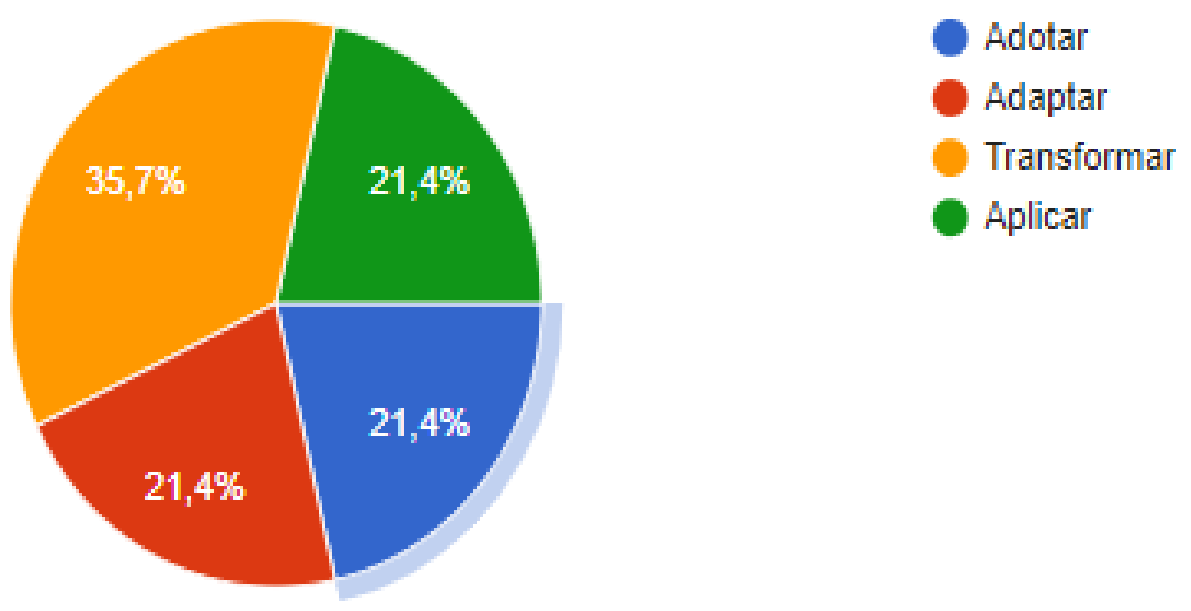

Fonte: Autores (2020).

Os participantes foram questionados com relação a aprendizagem em todo tempo e lugar e como gerenciam essa realidade, na questão "A educação digital possibilita o acesso à informação e ao conhecimento em diferentes espaços, contextos e temporalidades, tanto em ambientes formais de educação como não formais, na perspectiva da aprendizagem onlife 5 . Como tem gerido este momento na sua vida?".

As respostas se concentraram nas grandes possibilidades que a internet oferece às pessoas, mas também ao cuidado que precisamos ter e ensinar aos estudantes, sobre a veracidade e a confiabilidade das informações, conforme apresentado em algumas respostas a seguir.

\footnotetext{
"Atualmente a informação está disponível a distância de um clique, porém nem tudo que está na rede tem qualidade. Dessa forma, mesmo que o estudante encontre tudo o que busca, é necessário que $\circ$ professor $\circ$ auxilie nesse processo, para que encontre a
}

\footnotetext{
5 "O Manifesto Onlife - Ser humano em uma era hiperconectada", de 2015. The Onlife Manifesto Being Human in a Hyperconnected Era
} 
informação correta e de qualidade, de modo que seja possível transformá-la em conhecimento significativo." (Participante 10)

"A partir da adoção de práticas on-line em minha ação educativa como professora (tutora), e também como aluna de um curso de especialização totalmente online. Explorar a construção de novos saberes por meio do ambiente virtual é uma possibilidade que favorece o aprendizado." (Participante 2)

"É preciso disciplina e filtro para ter foco e se concentrar no que é essencial para o momento a fim de obter clareza sobre o que pode ser desenvolvido a médio e longo prazo." (Participante 6)

"Integrando aos demais formatos e interfaces, compreendendo a multirreferencialidade." (Participante 7)

A gestão do conhecimento é uma das competências essenciais para o século XXI, de acordo com o documento gerado na Conference Board of Canada, 2014, como ratifica Santos (2028, p. 185-187) ao refletir sobre o tema apresentado por, Bates (2016, p. 60-62),

O conhecimento não só está mudando rapidamente com as novas pesquisas, novos desenvolvimentos e rápida disseminação de ideias e práticas por meio da internet, mas as fontes de informação também estão aumentando, com uma grande variabilidade na confiabilidade ou validade das informações. Assim, o conhecimento [...] pode se tornar obsoleto. [...] A habilidade fundamental em uma sociedade baseada no conhecimento é a gestão do conhecimento: como encontrar, avaliar, analisar, aplicar e divulgar informações em um contexto particular. Esta é uma habilidade que os graduados precisarão empregar muito tempo depois da formatura.

Os participantes foram questionados a respeito dessa competência, "Cada vez mais é necessário que as pessoas estejam formadas para a gestão do conhecimento que envolve a aprendizagem ao longo da vida, o ser autônomo, o saber, o pesquisar, o refletir, o analisar e criar soluções possíveis. Escreva três palavras que podem revelar como tem gerido o seu conhecimento".

As respostas foram organizadas em uma nuvem, apresentada a seguir, que revela palavras com maior incidência; as menores, as palavras sinônimas, foram agrupadas. Podemos perceber que a pesquisa, 
acompanhada do compartilhamento e a necessidade de uma educação continuada, fortalece o valor da gestão do conhecimento em todo tempo.

Figura 6: Nuvem de palavras

\section{Compartilhamento Internet Pesquisa Inovar \\ Conhecer Educação continuada Inteligência emocional}

Fonte: Autores (2020).

Com relação ao questionamento "Acredita que utilizar estratégias didáticas diferenciadas amplie as possibilidades de aprendizagem?", todos os participantes revelaram que acreditam na mudança de paradigma educacional visando o maior protagonismo dos estudantes, com alcance de níveis cognitivos mais altos, saindo do modelo de memorização apenas, envolvendo mais análise, criação, cooperação, autonomia, colaboração, gestão de tempo, entre outras possibilidades de desenvolvimento dos estudantes e professores, características das estratégias didáticas diferenciadas. Percebe-se também a unanimidade de relação das escolhas das estratégias com a possibilidade de ampliação da aprendizagem.

A reflexão sobre RRI possibilitou que os participantes pudessem registrar suas perspectivas com relação a RRI, na questão "Como a Pesquisa e 
Inovação Responsáveis (Responsible Research and Innovation - RRI) pode ampliar as possibilidades de aprendizagens?".

Entre as respostas registradas podemos destacar que todos os participantes acreditam que as propostas de RRI possam envolver mais os estudantes, inserindo-os em seu contexto e principalmente possibilitando que ações individuais e coletivas tragam mudanças sociais, valorizando a pesquisa e a construção coletiva. A seguir, apresentam-se alguns textos que corroboram com esta afirmação.

"Por meio de atividades pautadas na abordagem RRI, haverá mais envolvimento e participação ativa do estudante na resolução de problemas, o desenvolvimento de um pensamento crítico, irá aprender a estimar riscos e consequências das ações na sociedade, a interrogar as fontes, não acreditar em tudo o que lê." (Participante 11)

"Envolvendo diferentes atores no processo da busca de soluções para problemas reais e da busca responsável do conhecimento por meio da pesquisa colaborativa." (Participante 12)

"Por meio da reflexão crítica, não somente sobre a temática, mas também na forma de pesquisa." (Participante 13)

"Pode ampliar as possibilidades de aprendizagens pesquisando coletivamente e individualmente. Estimular interesses em novo descobrimento." (Participante 14)

Podemos dizer que ações são base para uma boa proposta de RRI, como as que aparecem na questão "Como pesquisar, refletir, experimentar e criar, a partir de situações-problema reais, pode envolver os estudantes e os professores?". Os participantes retrataram a importância dos contextos e das interações como elementos relevantes para uma aprendizagem significativa. Em seus estudos, Santos (2018, p.59) cita que,

Neste momento histórico, fica evidente que o conhecimento também é propagado pelos estudantes e que entre eles a aprendizagem pode acontecer de forma significativa, com a interlocução qualificada, ou seja, a interação, o planejamento e a mediação, de um docente, podendo influenciar a comunidade e o contexto em que estão inseridos. 
Para dar sustentação a este tema específico, Torres (2014, p. 100) indica a importância de que,

[...] o educador passe a adotar novas metodologias de ensino e aprendizagem, pois a construção de conhecimentos necessita ser significativa e aplicável à realidade social e profissional do indivíduo, levando em consideração seus saberes já consolidados, integrando pessoa, sociedade, trabalho e natureza.

\author{
Destacam-se alguns trechos interessantes sobre a percepção dos \\ participantes da pesquisa com relação a todo o processo em contextos \\ reais.
}

"Ao adotar metodologia de ensino por meio de situações-problema estimulamos os estudantes a compartilhar dúvidas e descobertas, além disso, é possível colocar em destaque situações reais, visando assim envolver os discentes para analisar, refletir e propor soluções possíveis, aproximando-os da consolidação de uma aprendizagem significativa." (Participante 2)

"Situações-problema reais tornam o aprender com significado e leva os estudantes a investigar e querer descobrir soluções para tal. Envolve os estudantes, pois para solucionar o problema é preciso encontrar caminhos, pesquisar, experimentar e isso incentiva o processo de aprendizagem." (Participante 4)

"Práticas pedagógicas significativas por meio de projetos com temáticas relevantes pode envolver sim alunos e professores." (Participante 5)

"O professor deve atuar como mediador no processo de ensino e aprendizagem, auxiliando o estudante a transformar a informação disponível em conhecimento significativo. E com as situaçõesproblema reais, o estudante poderá analisar, discutir e tomar decisões compreendendo na prática como solucionar problemas complexos, pensando sempre na responsabilidade dos atos e ações na sociedade." (Participante 10)

"Envolve à medida que possibilita a leitura de contextos próximos, traz sentido ao que está sendo estudado pois ambos percebem a importância do porquê fazer." (Participante 14) 
Na questão "Descreva em 4 palavras como foi o processo de construção do curso de RRI para a formação de professores que realizou durante o grupo de pesquisa PUCPR" um cenário muito interessante é apresentado, em que $80 \%$ dos respondentes trouxe o elemento de desafio, o que significa que não é algo de seu cotidiano, chegando a aparecer as palavras "aflito e difícil".

A respeito de ser essa uma proposta que gera colaboração, interação e cooperação, ou seja, que possibilita aos agentes estar em constante ação e aprendizagem, $90 \%$ dos respondentes sinalizaram essa característica do projeto desenvolvido.

As ideias de necessidade de reflexão, caracterizando um processo criativo e cocriativo também apareceram nas respostas dos participantes, escritas com palavras sinônimas, em uma proporção significativa.

Para que essa reflexão proporcione uma visão ampla sobre a construção dos módulos do curso de RRI, apresenta-se a seguir o conjunto recursos criados para que os professores que terão acesso ao curso possam contar com diferentes oportunidades de aprendizagem.

QUADRO 3 - Recursos desenvolvidos para o MOOC de RRI

\begin{tabular}{|c|c|}
\hline Quebra-cabeças & $\begin{array}{c}\text { Levantamento de aplicativos, infográficos, } \\
\text { revistas etc. }\end{array}$ \\
\hline Questionário de sondagem & llustrações das etapas \\
\hline $\begin{array}{l}\text { Storytelling com recursos de } \\
\text { imagem e som }\end{array}$ & Tutorial para mapa mental de conteúdo \\
\hline Vídeos & Textos e atividades \\
\hline $\begin{array}{l}\text { Questionário no Google para } \\
\text { avaliar a relevância do curso }\end{array}$ & $\begin{array}{c}\text { Um jogo de cards para conhecimento e } \\
\text { instrução do uso das diversas ferramentas } \\
\text { tecnológicas na educação }\end{array}$ \\
\hline Duração: 2 meses & Carga horária: 40 horas \\
\hline
\end{tabular}




\begin{tabular}{|c|c|}
\hline $\begin{array}{l}\text { Uma apresentação em vídeo } \\
\text { das estratégias de ensino- } \\
\text { aprendizagem }\end{array}$ & $\begin{array}{c}\text { Ambiente: o curso foi desenvolvido no } \\
\text { Google Sala de Aula } \\
\text { https://classroom.google.com/ }\end{array}$ \\
\hline $\begin{array}{c}\text { Inscrições: } \\
\frac{\text { https://www.sympla.com.br/ }}{\text { curso-rri--uma-nova- }} \\
\text { estrategia-didatica } \quad 852834\end{array}$ & $\begin{array}{l}\text { Texto de divulgação: Curso de } 40 \text { horas, de } \\
\text { formação continuada on-line e aberto, } \\
\text { destinado a estudantes de licenciatura, a fim } \\
\text { de promover a formação, a disseminação e } \\
\text { a implementação de novos modos de } \\
\text { produzir ciência e de realizar a prática } \\
\text { docente, por meio conceito de Pesquisa e } \\
\text { Inovação Responsáveis (RRI). } \\
\text { Pretende desenvolver as } 10 \text { novas } \\
\text { habilidades científicas que compõem a RRI, } \\
\text { aliadas ao uso consciente e responsável de } \\
\text { meios e tecnologias digitais de produção e } \\
\text { divulgação do conhecimento produzido por } \\
\text { meio do REA (Recursos Educacionais } \\
\text { Abertos). }\end{array}$ \\
\hline
\end{tabular}

Fonte: Autores (2020).

Os professores, hoje, vivenciam um momento de total transformação, pois as demandas da educação digital exigem novas competências, como apresentado nessa reflexão. Quando instigados a desenvolver um curso a distância, MOOC, sobre um tema muito atual, que seria a RRI, algumas dificuldades tiveram que ser superadas. Dessa forma, acreditamos ser importante destacar o que os participantes escreveram, a fim de tornar evidente que todos os agentes envolvidos nos processos educacionais precisam ser resilientes e perceber a necessidade da gestão do seu conhecimento, mantendo a premissa de aprendizagem ao longo da vida.

$\mathrm{Na}$ questão "Descreva quais as dificuldades que vivenciou ao participar da construção do curso de formação de professores RRI", a maior 
parte das respostas não revelou problemas em criar objetos de aprendizagem, em pensar em uma estrutura diferenciada para curso on-line e nem mesmo em aprender novas ferramentas digitais, mas na própria temática do curso como observa-se na resposta a seguir, que explicita a importância dessa reflexão.

"Por se tratar de um tema ainda em construção, a inexistência de referenciais em português e mesmo internacional, dificultou um pouco $O$ entendimento e a aplicabilidade no processo educacional." (Participante 5)

Com relação ao que a proposta de construção do MOOC proporcionou, na questão "Descreva os pontos positivos da vivência da criação do curso durante o grupo de pesquisa", percebemos que os participantes puderam experienciar os dois elementos relevantes, a pesquisa e o compartilhamento, como demonstram as respostas a seguir.

\footnotetext{
"Descobertas, mais estudo do tema e o trabalho em equipe." (Participante 1)

"A oportunidade de compartilhar as dúvidas provocou aproximação entre os indivíduos do grupo e a receptividade no auxílio da construção dos materiais." (Participante 2)

"Todo o processo desenvolvido foi de grande valia, aprender pesquisando, adotando as tecnologias e adaptando as necessidades, logo, transformar o contexto do ser humano por meio da conscientização e humanização. O trabalho em equipe. $O$ companheirismo entre todos. A busca pela qualidade do trabalho que compõe o curso. O cuidado com a ética e a preocupação com a produção científica." (Participante 5)
}

Os momentos das reuniões do grupo de pesquisa e o Projeto de RRI no qual todos estão envolvidos possibilitaram muitos momentos de aprendizagem, destacados, por exemplo, na fala de um dos participantes ao responder que "Aprender com os pares è sempre instigante, nos traz novos desafios". 


\section{CONSIDERAÇÕES FINAIS}

O tema RRI tende a ser continuamente estudado, pois a pesquisa responsável pode ser uma das grandes estratégias didático-pedagógicastecnológicas com o objetivo de colaborar com o desenvolvimento de professores e estudantes.

A proposta deste curso pretende expandir-se após a aplicação nas primeiras turmas, e com possíveis ajustes baseados nos feedbacks dos estudantes que responderão a um questionário e terão a oportunidade de contar suas expectativas e resultados obtidos.

Como citou um dos participantes, sabemos que "O professor deve atuar como mediador no processo de ensino e aprendizagem, auxiliando o estudante a transformar a informação disponível em conhecimento significativo"; as propostas de RRI promovem essa condição.

Percebemos também, como citado por um dos respondentes, que o contexto real amplia as possibilidades de engajamento e que "o estudante poderá analisar, discutir e tomar decisões compreendendo na prática como solucionar problemas complexos, pensando sempre na responsabilidade dos atos e ações na sociedade", o que auxilia no desenvolvimento da cidadania e na construção de pessoas mais engajadas, comprometidas e disponíveis para enfrentar os desafios deste século.

Com relação às questões sobre educação, as propostas de RRI tendem a apoiar o desenvolvimento do pensamento científico, oportunizando para professores e estudantes um espaço para o crescimento de conhecimentos a respeito da pesquisa, apoiando a problematização, a criação de hipóteses, a resolução de problemas e a criação de soluções efetivas para problemas reais envolvendo a comunidade e os contextos. 


\section{REFERÊNCIAS}

AZINHAGA, Patrícia Fialho; MARQUES, Ana Rita; REIS, Pedro. Investigação e Inovação Responsáveis em contexto educativo: percepções de estudantes e professores quanto às potencialidades e limitações das atividades prepostas no âmbito do projeto IRRISISTIBLE. 2016, Lisboa, Espanha. Disponível em: https://repositório.ul.pt>handle. Acesso em: dez. 2019.

BATES, A. W. (Tony). Educar na era digital, design, ensino e aprendizagem. São Paulo. Artesanato educacional, 2016. Título original: Teaching in digital age, guidelines for designing teaching and learning.

CESE. La recherche et l'innovation responsables - Science et société civile travaillent de concert, 2016. Disponível em: https:www.eesc.europa.eu> larech Acesso em: nov. 2019.

DRAVET, Florence, PASQUIER, Florent, COLLADO, Javier, CASTRO, Gustavo de. Transdisciplinaridade e educação do futuro. Brasília: Cátedra UNESCO de Juventude, Educação e Sociedade; Universidade Católica de Brasília, 2019. 260 p.; 24 cm. ISBN: 978-85-62258-39-8. Disponível em:

<https://www.researchgate.net>3372> Acesso em: nov. 2019.

EUROPEAN COMMISSION. The education and training monitor. Luxembourg: Publications Office of the European Union, 2017.

FAVA, Rui. Educação 3.0. São Paulo: Saraiva, 2014.

FERNANDEZ, Beltran; GARCIA-MARZÁ, Domingo; SANAHUJA, Rosana; ANDRES MARTINEZ, Alicia; FORCADELL, SUsana Barberá. La gestion de la comunicacion para el impulso de la Investigacion e Innovacion Responsables: propuesta de protocolo desde la etica dialógica. 2017. Revista Latina de Comunicación Social, 72, pp.1.040 a 1.062, Espanha. Disponível em: <repositori.uji.es>handle>58> Acesso em: nov. 2019.

FLORIDI, L. (Ed.) The onlife manifesto: being human in a hyperconnected era. London: Springer, 2015. Disponível em:

<https://link.springer.com/content/pdf/10.1007\%2F978-3-319-04093-6.pdf> Acesso em: abr. 2016

HERRANZ, Margarita Blazquez. Investigacion e Innovacion Responsable. Una Aproximacion a la Investigacion en Salud, 2016. Disponível em: <https://ec.europa.eu>eip>files>. Acesso em: dez. 2019. 
HERRERA, Pablo Matias. Bonos de Impacto Social: de la Innovacion Social a la Innovacion Financiera Responsable, 2015, TRILOGIA. Ciencia. Tecnologia y Sociedade / vol. 7 Num.13ISSN 2145-4426 / julio-diciembre / 2015 / pp.63 - 73. Disponível em: https://dialnet.unirioja.es>art. Acesso em: nov. 2019.

HORIZON 2020 - O programa-quadro de investigação e inovação da UE. Luxemburgo: Serviço das Publicações Oficiais das Comunidades Europeias, 2014. ISBN 978-92-79-38928-3 doi:10.2777/82557 @ União Europeia, 2014. Disponível em:

<https://ec.europa.eu/programmes/horizon2020/sites/horizon2020/files/H2020 _PT_KI0213413PTN.pdf> Acesso em: nov. 2019.

OBSERVATORI DE BIOÉTICA I DRET, UNIVERSITAT DE BARCELONA, INVESTIGACION E INNOVACION RESPONSABLE, S. D., Bacelona, Espanha. Bioética y Derecho. Disponível em: <www. Bioeticayderecho.ub.edu>. Acesso em: dez.2019

PINTO, Sonia Maria da Conceição e Ribeiro, FERREIRA, Silvar. Pesquisa e Inovação Responsáveis na Formação Cientifica dos Estudantes da Educação Superior, 2018, São Paulo, Brasil. Revista e-curriculum, São Paulo, v. 16, n. 2, p. 420 - 444 abr./jun. 2018, programa de Pós-graduação Educação: Currículo - PUC/SP. Disponível em: <http://revistas.puesp.br/index.php/curricullum> Acesso em: nov. 2019.

REIS, Pedro, MARQUES Ana Rita. A Investigação e Inovação Responsáveis em sala de aula: Módulos de Ensino. IRRESITIBLE, 2016, Lisboa, Espanha. Disponível em: https://r positorio.ul.p>

SANTOS. Katia E. E. A educação híbrida no processo de ensinoaprendizagem: uma proposta norteadora. Tese de doutorado. Pontifícia Universidade Católica do Paraná. 508 p. 2018.

TORRES, Patricia, CARPIM, Lucymara, BEHRENS, Marilda Aparecida.

Paradigma da Complexidade na Prática Pedagógica do Professor de Educação Profissional no Século 21. B. Tec. Senac, Rio de Janeiro, v. 40, n.1, p. 90-107, jan./abr. 2014. Disponível em:

<file:///C:/Users/mauri/Downloads/1 15-234-1-SM.pdf> Acesso: jan. 2020.

Recebido em: 27 de fevereiro de 2020 Aprovado em: 14 de junho de 2020 Publicado em: 30 de julho de 2020

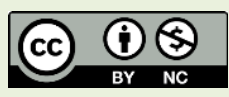

